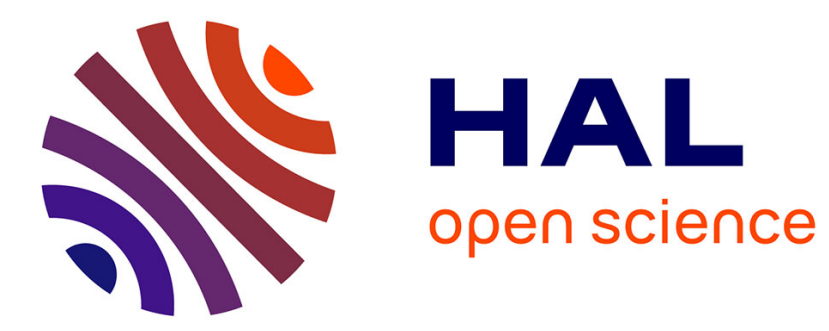

\title{
Results from NA57
}

H. Helstrup, F. Antinori, P.A. Bacon, A. Badala, R. Barbera, A. Belogianni, I.J. Bloodworth, M. Bombara, G. Bruno, S.A. Bull, et al.

\section{To cite this version:}

H. Helstrup, F. Antinori, P.A. Bacon, A. Badala, R. Barbera, et al.. Results from NA57. 8th International Conference of Strangeness in Quark Matter, Mar 2006, Los Angeles, United States. pp.S89-S95, 10.1088/0954-3899/32/12/S11 . in2p3-00102878

\section{HAL Id: in2p3-00102878 https://hal.in2p3.fr/in2p3-00102878}

Submitted on 4 Oct 2006

HAL is a multi-disciplinary open access archive for the deposit and dissemination of scientific research documents, whether they are published or not. The documents may come from teaching and research institutions in France or abroad, or from public or private research centers.
L'archive ouverte pluridisciplinaire HAL, est destinée au dépôt et à la diffusion de documents scientifiques de niveau recherche, publiés ou non, émanant des établissements d'enseignement et de recherche français ou étrangers, des laboratoires publics ou privés. 


\title{
Results from NA57
}

\author{
Håvard Helstrup ${ }^{1}$ for the NA57 Collaboration $\ddagger$ \\ ${ }^{1}$ Høgskolen i Bergen, Bergen, Norway
}

\begin{abstract}
The NA57 experiment has measured strange baryon and antibaryon production in $\mathrm{Pb}-\mathrm{Pb}$ collisions at $40 \mathrm{~A} \mathrm{GeV} / c$ and $158 \mathrm{~A} \mathrm{GeV} / c$ beam momenta. Expansion dynamics has been investigated; transverse dynamics using blast wave fits, longitudinal dynamics obtained from rapidity distributions. HBT analysis gives results compatible with those obtained by the other methods. Similar values are observed for the transverse and longitudinal flow parameters. Particles carrying all levels of strangeness have been studied. Enhancement factors increase with the strangeness content of the particle, reaching above 20 for $\Omega \mathrm{s}$ in central collisions. Enhancement values are strikingly similar for SPS and RHIC energies. Centralto-peripheral nuclear modification factors have been measured. The baryon/meson pattern displays similarities with that observed at RHIC.
\end{abstract}

\section{Introduction}

The NA57 experiment has been designed to study production of strange and multistrange particles in $\mathrm{Pb}-\mathrm{Pb}$ collisions at the CERN SPS. The experiment has extended previous WA97 [1] measurements to a wider centrality range and to lower beam momentum.

Enhanced production of strange particles was one of the first signatures to be proposed for the Quark-Gluon Plasma in heavy ion collisions [2]. The observed pattern of strangeness enhancement factors increasing with the strangeness content of the particle agrees with theoretical predictions describing particles originating from a dense system with interactions at the parton level.

The expansion dynamics of the dense system can be extracted from the measured final state particle spectra. Blast wave analysis has been applied to the transverse mass spectra to obtain kinetic freeze-out temperature and radial flow velocity. The longitudinal flow velocity has been obtained from fits to rapidity distributions.

RHIC data shows a significant suppression of high transverse momentum particles in central heavy ion collisions. This effect is quantified using nuclear modification factors, which are calculated as the ratio of yields per binary collision in nucleus-nucleus collisions compared to proton-proton collisions $\left(R_{A A}\right)$ or central to peripheral nucleus-nucleus collisions $\left(R_{C P}\right)$. The NA57 experiment has measured $R_{C P}$ factors for negatives and singly strange particles.

$\ddagger$ A list of all members of this collaboration is given at the end of this issue. 


\section{Experimental setup and data analysis}

The layout of the NA57 experiment has been described elsewhere [3]. The main tracking device is a silicon pixel telescope, with a $5 \times 5 \mathrm{~cm}^{2}$ cross section. Planes are densely stacked in the compact part of the telescope, with a total length of $30 \mathrm{~cm}$. Additional lever arm tracking detectors are used to improve the resolution for high momentum particles. Multiplicity is measured by two detector systems, a scintillator petal detector used for triggering and two planes of silicon microstrips used for offline analysis.

Strange particles are identified through their decays to charged particles. All decays are required to take place in a fiducial volume located between the target and the telescope, and all decay particles are required to pass through the compact part of the pixel telescope. Kinematic cuts are applied to identify strange particle decays.

The multistrange particle spectra are corrected by calculating a correction weight (which takes into account acceptance and efficiency effects) for each identified particle. The same procedure has been applied to the much more abundant singly strange particles, but in this case weights have been calculated only for a subsample of the measured particles, as in this case systematic errors are already the dominant contribution.

The collision centrality is determined from the charged particle multiplicity $\left(N_{c h}\right)$ using the multiplicity microstrip detectors as described in $[4,5]$. The $N_{c h}$-differential $\mathrm{Pb}-\mathrm{Pb}$ cross section $\mathrm{d} \sigma / \mathrm{d} N_{c h}$ is fitted assuming $N_{c h}=q \cdot N_{\text {part }}^{\alpha}$ [4] (a modified Wounded Nucleon model) where $N_{\text {part }}$ is the number of participants evaluated from the Glauber model and $q$ is a proportionality constant. The sample of collected events is divided into centrality classes. For each class the average number of participants $\left\langle N_{\text {part }}\right\rangle$ and binary collisions $\left\langle N_{c h}\right\rangle$ is calculated.

\section{Transverse dynamics: Blast wave fits}

Transverse mass spectra obtained for $\mathrm{K}_{\mathrm{S}}^{0}$ and for $\Lambda, \Xi^{-}, \Omega^{-}$and their antiparticles for the two beam momenta are shown in the leftmost two panels of figure $1[6,7] \S$ The $m_{\mathrm{T}}$ spectra have been analyzed within the blast wave model [8] assuming a linear velocity profile. The blast wave fits are shown as dotted lines in figure 1.

The resulting kinetic freeze-out temperature and average transverse flow velocity are:

$$
\begin{aligned}
& T=(118 \pm 5 \pm 11) \mathrm{MeV},\left\langle\beta_{\perp}\right\rangle=0.38 \pm 0.01 \pm 0.01 \text { at } 40 \mathrm{~A} \mathrm{GeV} / c \\
& T=(144 \pm 7 \pm 14) \mathrm{MeV},\left\langle\beta_{\perp}\right\rangle=0.40 \pm 0.01 \pm 0.01 \text { at } 158 \mathrm{~A} \mathrm{GeV} / c
\end{aligned}
$$

Comparing more central to more peripheral collisions, the temperature parameter decreases whereas the transverse flow velocity increases. Comparing $40 \mathrm{~A} \mathrm{GeV} / c$ results to those obtained at $158 \mathrm{~A} \mathrm{GeV} / c$, the kinetic freeze-out temperature is lower and the transverse flow velocity is similar, as shown in the right panel of figure 1.

$\S$ See therein for original references to the various NA44, NA49 and CERES data used to compile these figures. 

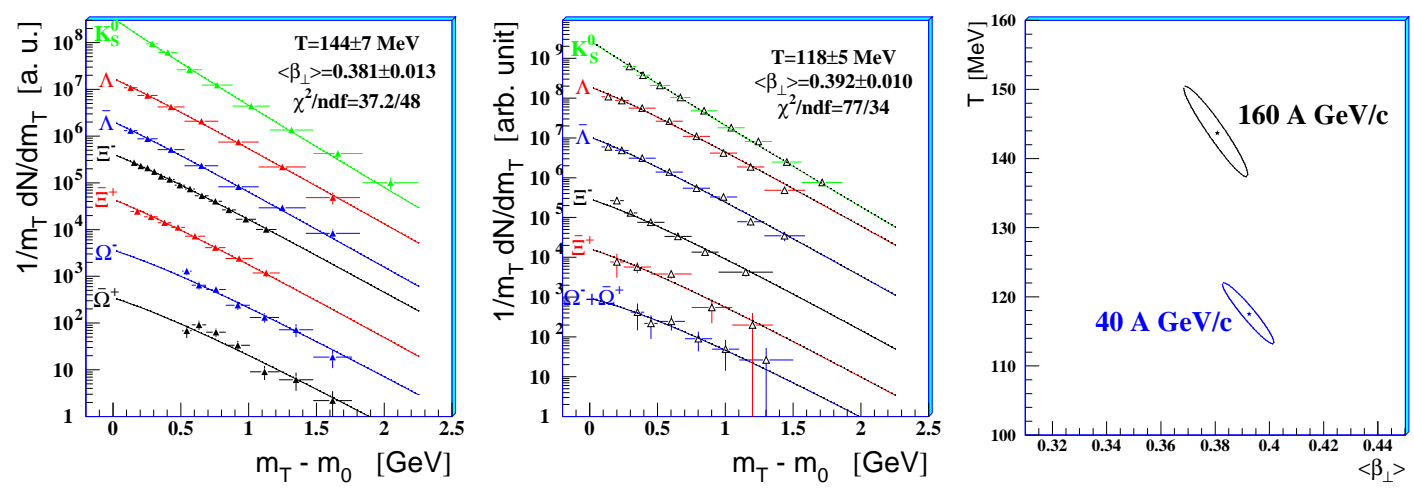

Figure 1. Blast-wave fits to transverse mass spectra for the $53 \%$ most central collisions at 158 (left) and 40 (middle) $\mathrm{A} \mathrm{GeV/c}$. To the right is shown the $1 \sigma$ contour levels of the fit at the two beam momenta.
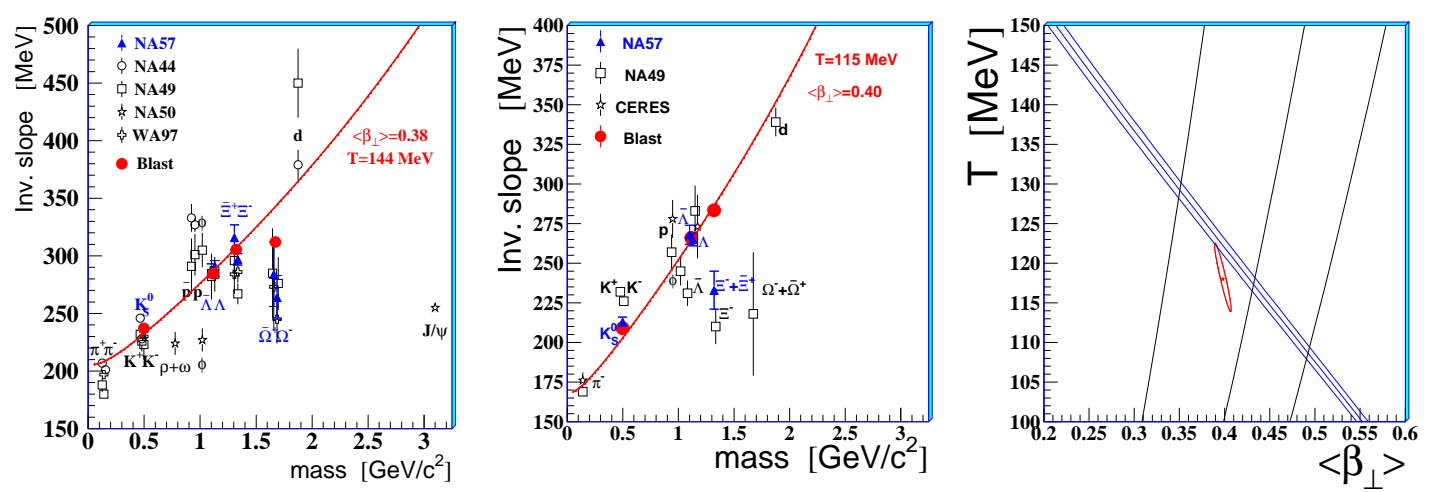

Figure 2. Inverse slope parameters for various particles with predictions of the blast wave model at $158 \mathrm{~A} \mathrm{GeV} / c$ (left) and $40 \mathrm{~A} \mathrm{GeV} / c$ (middle). The right panel shows temperature and transverse flow parameters obtained by three independent methods from NA57 data.

The inverse slope parameters are plotted as a function of particle mass at the two beam momenta in figure 2 . The full line corresponds to the inverse slope which would be obtained for a generic particle of mass $m_{0}$ using the blast wave fit parameters obtained from the best fit to singly strange particles at the corresponding beam momentum. Since the inverse slope is a function of the $m_{\mathrm{T}}-m_{0}$ range where the fit is performed, the inverse slopes of the particles identified by NA57 has also been computed within the $m_{\mathrm{T}}-m_{0}$ range of NA57. These values are shown by full circles in figure 2 . The multiply strange particles clearly deviate from the blast wave fit, and are better described by a fit with higher temperature and lower transverse flow velocity, suggesting earlier decoupling of these particles.

The right hand panel of figure 2 shows temperature and transverse flow parameters obtained from the NA57 data at $40 \mathrm{~A} \mathrm{GeV} / c$ using three independent methods. The lines with a negative slope (blue in the online version) are obtained by applying the 
blast wave model to the spectrum of negative particles, the lines with a positive slope (black) from HBT analysis and the contour plots at the centre (red) from the blast wave analysis of singly strange particles. The three lines of each group show the central value and the one sigma error band. A very good overlap from all three methods at the values quoted above from the blast wave fit is observed.

\section{Longitudinal dynamics: Rapidity distributions}

Rapidity distributions for the $158 \mathrm{~A} \mathrm{GeV} / c$ data sample have been obtained for all strange particles identified by NA57 [9]. Within the NA57 acceptance region, all distributions are compatible with being flat, except for $\mathrm{K}_{\mathrm{S}}^{0}$ and $\bar{\Lambda}$, for which a Gaussian shape is clearly visible. The rapidity distributions have been used to extract information about the longitudinal expansion of the system, using the blast wave model of Bjorken [10] and Landau [11] hydrodynamics folded with a thermal distribution of fluid elements $[8,12]$.

The rapidity distributions with the fit including longitudinal flow are shown in figure 3. The extracted longitudinal flow velocity is $\left\langle\beta_{L}\right\rangle=0.42 \pm 0.03$, which is rather similar to the transverse flow velocity discussed above. This may indicate a significant stopping of the incoming nuclei at the top SPS energy, and may indicate an almost isotropic expansion of the fireball produced in the collision.

\section{Strangeness enhancements}

The observed cross section of each particle species has been fitted by the expression

$$
\frac{\mathrm{d}^{2} N}{\mathrm{~d} m_{\mathrm{T}} \mathrm{d} y}=f(y) m_{\mathrm{T}} \exp \left(-\frac{m_{\mathrm{T}}}{T}\right) .
$$

The rapidity distribution is taken to be flat within our acceptance region for all particles except for $\mathrm{K}_{\mathrm{S}}^{0}$ and $\bar{\Lambda}$, where a Gaussian distribution was used. The central rapidity yield is obtained by extrapolating to a common phase space region covering the full $m_{\mathrm{T}}$ range and one unit of rapidity around mid rapidity:

$$
Y=\int_{m}^{\infty} \mathrm{d} m_{T} \int_{y_{c m}-0.5}^{y_{c m}+0.5} \frac{\mathrm{d}^{2} N}{\mathrm{~d} m_{T} \mathrm{~d} y} \mathrm{~d} y .
$$

The enhancement factor $\mathrm{E}$ is defined as the yield per participant in each multiplicity bin divided by the similar yield obtained in proton-beryllium interactions:

$$
E=\left(\frac{Y}{\left\langle N_{\text {part }}>\right.}\right)_{\mathrm{Pb}-\mathrm{Pb}} /\left(\frac{Y}{\left\langle N_{\text {part }}\right\rangle}\right)_{\mathrm{p}-\mathrm{Be}} .
$$

The $158 \mathrm{~A} \mathrm{GeV} / c$ results are shown in figure 4. The hierarchy of enhancements, with $\mathrm{E}(\Lambda)<\mathrm{E}(\Xi)<\mathrm{E}(\Omega)$ is consistent with early predictions of strangeness production in a Quark Gluon Plasma scenario [2]. The enhancement factors reach above 20 for $\Omega$ in 

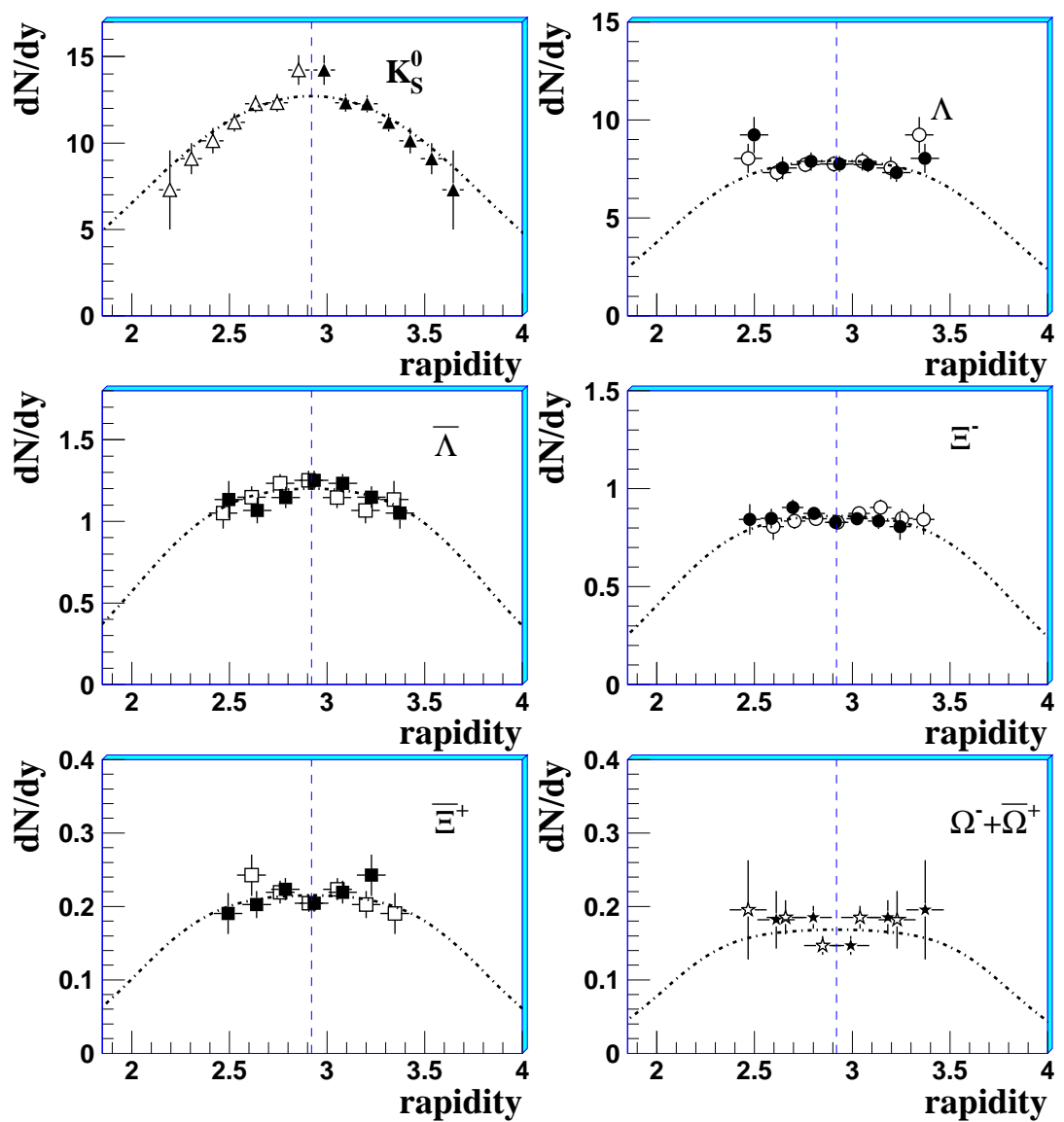

Figure 3. Rapidity distributions of strange particles in the full centrality range of NA57 (53\%). The full lines shows a fit by a thermal model including longitudinal flow.
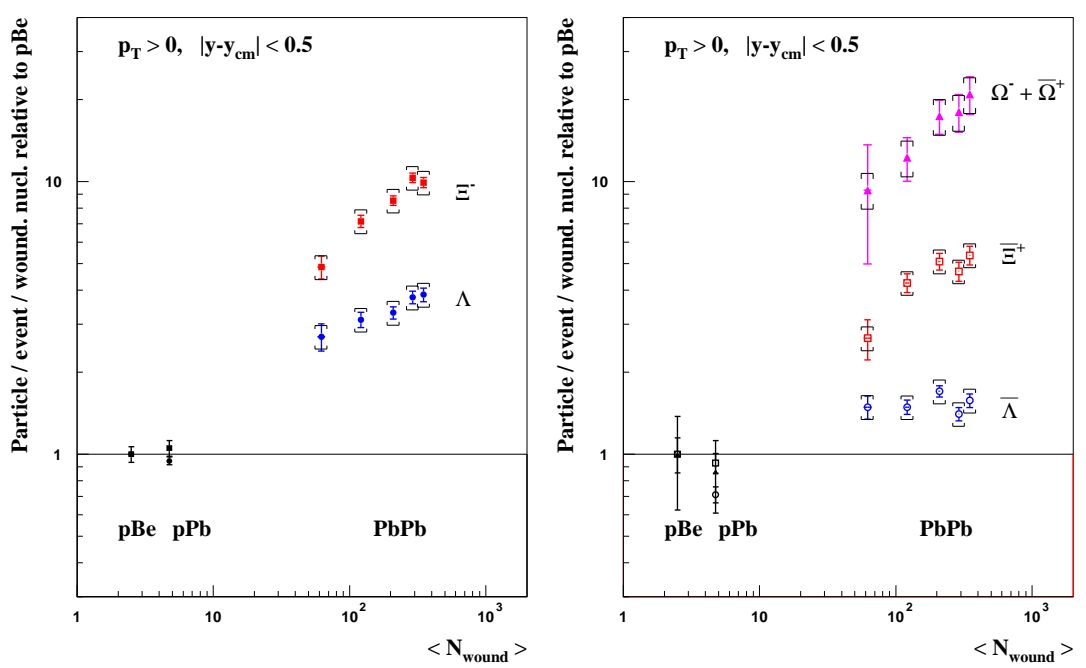

Figure 4. Centrality dependence of hyperon enhancements observed at $158 \mathrm{~A} \mathrm{GeV} / c$. The left panel shows particles having at least one quark in common with the incoming nuclei, the right panel shows particles containing quarks created in the collision only. 

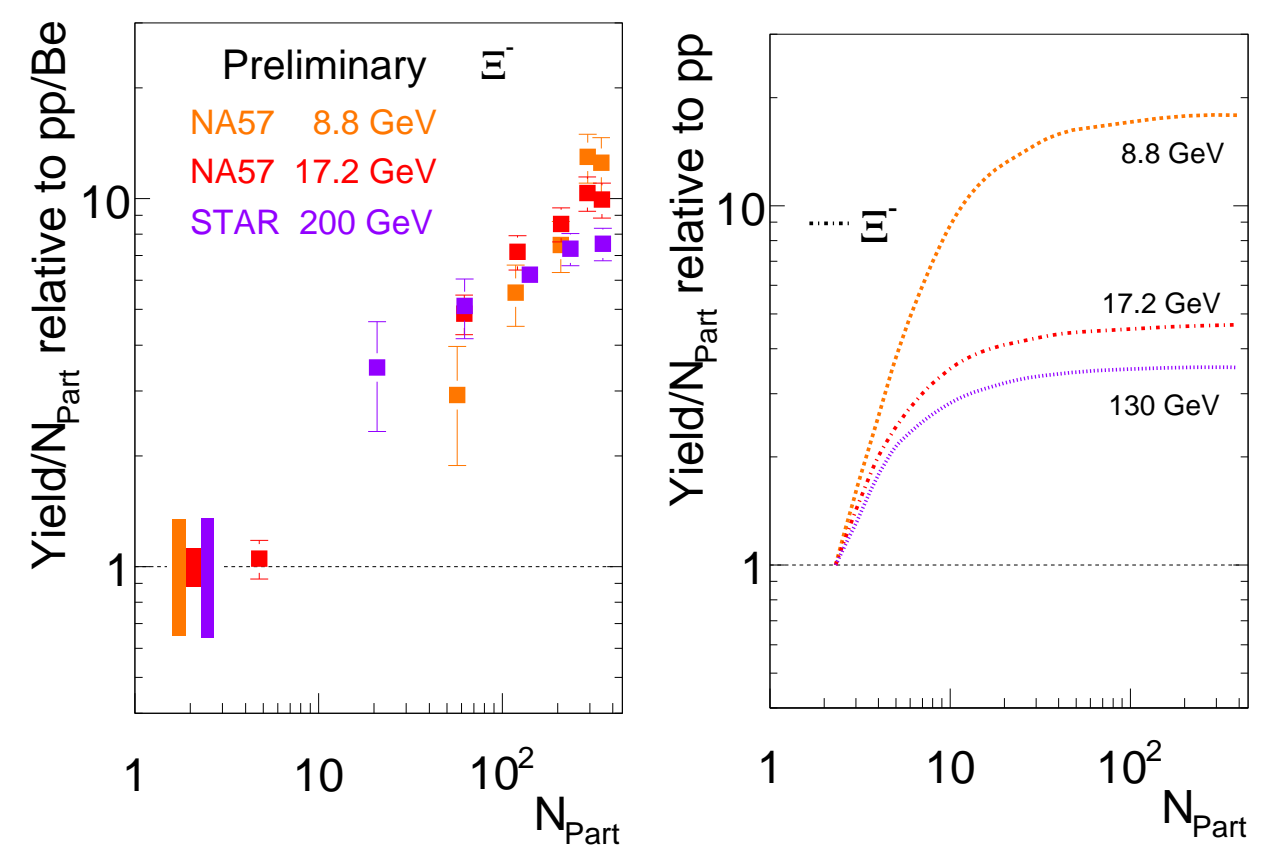

Figure 5. Enhancement of $\Xi^{-}$at different energies. The left panel shows measured data by NA57 and STAR [14], the right panel shows canonical suppression calculations [13].

the most central bins. For all particles except $\bar{\Lambda}$ there is a clear centrality dependence. A saturation behaviour in the two most central bins cannot be excluded.

The enhancements observed at $40 \mathrm{~A} \mathrm{GeV/c}$ are rather similar (due to low statistics in our proton-beryllium sample, enhancements for $\bar{\Xi}^{+}$and $\Omega$ cannot be obtained at 40 A GeV/c). Calculations based on the Canonical Suppression Model [13] suggested a clear energy dependence of the enhancement factors, with decreasing enhancements for increasing beam momenta. The enhancements are found to be rather similar for all observed energies, both at SPS and RHIC, as confirmed by recent STAR data [14]. A slightly steeper centrality dependence can be observed for the data at lower energy, as displayed in figure 5.

\section{Nuclear modification factors}

Nuclear modification factors have been used by the RHIC experiments to quantify nuclear effects. The central-to-peripheral nuclear modification factor is defined as

$$
R_{C P}\left(p_{\mathrm{T}}\right)=\frac{\left\langle N_{\text {coll }}\right\rangle_{\mathrm{P}}}{\left\langle N_{\text {coll }}\right\rangle_{\mathrm{C}}} \times \frac{\mathrm{d}^{2} N_{A A}^{C} / \mathrm{d} p_{\mathrm{T}} \mathrm{d} y}{\mathrm{~d}^{2} N_{A A}^{P} / \mathrm{d} p_{\mathrm{T}} \mathrm{d} y} .
$$

A suppression of $R_{C P}$ at high $p_{\mathrm{T}}$ is observed by the RHIC experiments (see e.g. [15]). Also a markedly different behaviour between baryons and mesons has been seen, confirmed to be a particle type effect rather than a mass effect by the measurement of the nuclear modification factor of the $\phi$ particle [15]. 

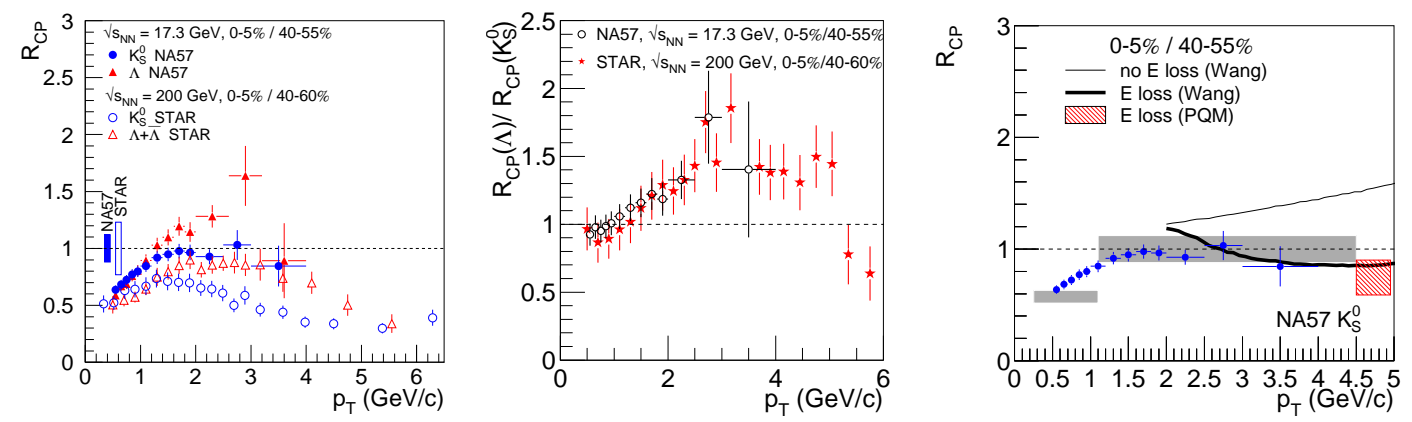

Figure 6. Left: Nuclear modification factors observed at SPS and RHIC. NA57 points shown with filled symbols, STAR points with open symbols. Middle: Relative $\mathrm{K}_{\mathrm{S}}^{0}$ and $\Lambda R_{C P}$ factors at SPS (open points) and RHIC (filled stars). Right: Model comparisons to NA57 $R_{C P}$ data. Thin line without energy loss in the medium, thick line including energy loss.

The NA57 experiment has measured nuclear modification factors for negatives, $\mathrm{K}_{\mathrm{S}}^{0}$, $\Lambda$ and $\bar{\Lambda}$, thus covering both mesons and baryons carrying one unit of strangeness. The results are shown in the left hand panel of figure 6 , along with similar ratios obtained by the STAR experiment at RHIC [16]. No suppression effect at high $p_{\mathrm{T}}$ is observed at SPS. Nevertheless the relative pattern of the $\mathrm{K}_{\mathrm{S}}^{0}$ and $\Lambda R_{C P}$ factors is strikingly similar between NA57 data from SPS and STAR data from RHIC. At RHIC this meson/baryon ratio has been interpreted as due to recombination effects. Recent $62 \mathrm{~A} \mathrm{GeV} / c$ data from RHIC [17] confirms this pattern.

The NA57 $R_{C P}$ data has also been compared to model predictions by X.N. Wang $[18,19]$, as shown in figure 6 . The calculation including parton energy loss in the medium (thick line) describes the NA57 data better than the calculations without energy loss. Also a PQM [20] calculation with energy loss (dashed area in the right panel of figure 6) is consistent with the NA57 measurement.

\section{Conclusions}

The expansion of the dense medium produced in lead-lead collisions at SPS has been described by a hydrodynamic model, yielding similar values for the transverse and longitudinal expansion velocities, suggesting a rather isotropic expansion profile. At $40 \mathrm{~A} \mathrm{GeV} / c$ a similar transverse flow velocity, but a lower temperature parameter is observed as compared to $158 \mathrm{~A} \mathrm{GeV/c}$.

Strangeness enhancements have been observed at both beam momenta. The enhancement factors increase with increasing strangeness content of the particle in both cases, in agreement with predictions from QGP models.

Central-to-peripheral nuclear modification factors have been measured for negatives and particles carrying one unit of strangeness. The ratio between $\mathrm{K}_{\mathrm{S}}^{0}$ and $\Lambda R_{C P}$ is very similar at SPS and RHIC energies, within the $p_{\mathrm{T}}$ range covered by NA57. 


\section{References}

[1] Andersen Eet al 1999 Phys. Lett. B449 401

[2] Rafelski J and Müller B, 1982, Phys. Rev. Lett. 481066

Rafelski J and Müller B, 1986, Phys. Rev. Lett. 562334

[3] Virgili T et al 2001 Nucl. Phys. A681 165c Antinori F et al 2006 J. Phys. G: Nucl. Part. Phys. 32427

[4] Antinori F et al 2000 Eur. Phys. J. C18 57

[5] Antinori F et al 2005 J. Phys. G: Nucl. Part. Phys. 31321

[6] Antinori F et al 2004 J. Phys. G: Nucl. Part. Phys. 30823

[7] Bruno G E et al 2005 J. Phys. G: Nucl. Part. Phys. 31 S127

[8] Schnedermann E, Sollfrank J, Heinz U 1993 Phys. Rev. C48 2462

[9] Antinori F et al. 2005 J. Phys. G: Nucl. Part. Phys. 311345

[10] Bjorken J D 1983 Phys. Rev. D27 140

[11] Landau L D 1953 Izv. Akad. Nauk. SSSR 1751 Belenkij S and Landau L D 1955 Usp. Fiz. Nauk 56309

Belenkij S and Landau L D 1956 Nuovo Cimento (suppl.) 315

[12] Mohanty B and Alam J 2003 Phys. Rev. C68 064903

[13] Redlich K and Tounsi A 2002 Eur. Phys. J C24 589

[14] Caines H 2005 Quark Matter 2005 proceedings, to be published, nucl-ex/0601014

[15] Dunlop J 2006 Nucl. Phys. A Quark Matter 2005 proceedings, to be published

[16] Adams J et al 2004 Phys. Rev. Lett. 92052302

[17] Caines H 2006 these proceedings

[18] Wang X N 2000 Phys. Rev. C61 064910

Wang X N 1998 Phys. Rev. Lett. 812655

Wang X N 2004 Phys. Lett. B595 165

[19] Wang X N 2005 private communication

[20] Dainese A, Loizides C, Paić G 2005 Eur. Phys. J. C38 461; private communication 